\title{
BCG vaccination: An update on current Australian practices
}

CPD

Jemma Wittner Taylor, Nigel Curtis, Justin Denholm

\section{Background}

The Bacille Calmette-Guérin (BCG) vaccine is primarily used to prevent tuberculosis (TB) infection and disease in settings with high TB incidence.

\section{Objective}

The aim of this review article is to describe the current uses of BCG vaccination in Australia, including the indications and contraindications, efficacy and other off-target effects, and the role of the general practitioner.

\section{Discussion}

$B C G$ vaccination in Australia is primarily used to prevent TB in neonates and children with a high risk of TB exposure. The BCG vaccine is most effective at preventing severe disseminated TB disease in young children and has variable efficacy in preventing adult disease. The BCG vaccine is usually well tolerated; however, given the small risk of adverse effects, vaccination should be undertaken by a practitioner experienced in its administration. When indicated, the $B C G$ vaccine is available from specialist TB or travel medicine centres.
WHILE BACILLE CALMETTE-GUÉRIN (BCG) is among the oldest and most widely used vaccines globally, it is not routinely used in Australia because of the low prevalence of tuberculosis (TB). As a result, many practitioners have limited experience with the use and administration of this vaccine. In recent years, there has been increasing interest in BCG vaccination for both established and novel indications, including protection against COVID-19. The aim of this article is to review BCG vaccination in the Australian context, including consideration of potential therapeutic uses beyond prevention of mycobacterial infections. Some key definitions are outlined in Table 1.

\section{What is the $B C G$ vaccine?}

The BCG vaccine was developed in the early 1900 s to protect against Mycobacterium tuberculosis. ${ }^{1,2}$ It is a live-attenuated strain of M. bovis, ${ }^{3}$ and multiple strains exist, manufactured in different countries. ${ }^{4}$ When used as a vaccine to protect against $\mathrm{TB}$, it is given intradermally. ${ }^{1}$

Globally, BCG is one of the most widely administered vaccines. It is a major part of TB control programs in areas with high TB incidence. ${ }^{5}$ It has a significant role in preventing TB disease, especially

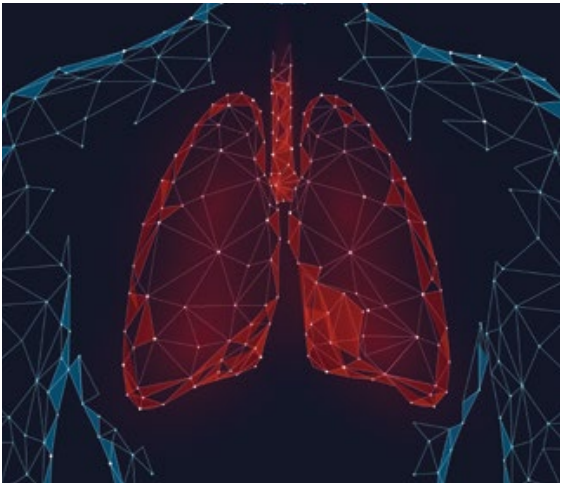

disseminated $\mathrm{TB}$ and $\mathrm{TB}$ meningitis in young children. ${ }^{6}$

Prior vaccination with BCG can sometimes result in a positive tuberculin skin test (TST; also known as Mantoux test). This cross-reactivity does not occur with interferon gamma release assays (IGRAs). Testing for latent TB infection (LTBI) throughout Australia commonly uses either TSTs or IGRAs, depending on patient characteristics, clinician preference and local availability. TB IGRAs are widely available in Australia; however, the Medicare Benefits Schedule only provides a rebate in certain circumstances (ie immunosuppression, human immunodeficiency virus [HIV] infection, exposure to a confirmed case of active TB, renal dialysis or silicosis).

\section{Indications}

The BCG vaccine was part of the Australian vaccination schedule until 1985 and was given to young adolescents through school-based programs. ${ }^{7,8}$ As the incidence of TB decreased below five per 100,000 population, and the risk of TB decreased, the vaccine was removed from the schedule in line with the International Union Against Tuberculosis and Lung Disease guidelines. ${ }^{5}$

Current indications for BCG vaccination in Australia are listed in Table 2. Current 
recommendations focus on providing the BCG vaccine to neonates and young children at highest risk. These are children in some Aboriginal and Torres Strait Islander communities, children travelling to high-incidence countries, ${ }^{9}$ and household contacts of people with Hansen's disease (leprosy). ${ }^{1}$ BCG vaccination is only recommended for those with occupational exposure if they have a high risk of exposure to drug-resistant TB, such as laboratory workers directly involved in culture of multi-drug resistant (MDR) TB.
The vast majority of clinical staff working in Australian healthcare settings do not require $B C G$ vaccination.

BCG is not routinely recommended as a pre-travel vaccine for adults. However, for specific high-risk travellers (eg medical or nursing staff working with MDR TB programs internationally), specialist advice at a dedicated TB service should be sought.

BCG vaccination is not recommended for family members of people undergoing treatment for active or latent TB.

\section{Table 1. Abbreviations and terms used}

\begin{tabular}{ll}
\hline Abbreviation/term & Meaning \\
\hline TST & Tuberculin skin test, also known as the Mantoux test \\
& Tuberculin (protein derived from Mycobacterium \\
& tuberculosis) is injected intradermally and the site \\
& monitored to establish if there is immune recognition of \\
& tuberculin suggesting prior exposure
\end{tabular}

\begin{tabular}{ll}
\hline IGRA & $\begin{array}{l}\text { Interferon gamma release assay } \\
\text { Antigen is presented to the patient's blood in vitro to } \\
\text { measure interferon gamma release; response to the } \\
\text { antigen suggests immune recognition }\end{array}$ \\
\hline TB IGRA & $\begin{array}{l}\text { An IGRA using TB antigen to test for immune recognition } \\
\text { of TB, suggesting prior exposure }\end{array}$ \\
\hline LTBI & Latent tuberculosis infection \\
\hline MDR-TB & Multi-drug resistant TB \\
\hline Miliary TB & $\begin{array}{l}\text { A characteristic appearance on chest imaging suggestive } \\
\text { of disseminated tuberculosis disease }\end{array}$ \\
\hline
\end{tabular}

$T B$, tuberculosis

\section{Table 2. Current Bacille Calmette-Guérin vaccination indications in Australia ${ }^{1}$}

\begin{tabular}{ll}
\hline Population & Recommendations \\
\hline $\begin{array}{l}\text { Aboriginal and Torres Strait } \\
\text { Islander people }\end{array}$ & $\begin{array}{l}\text { Recommended for neonates in some areas of NT, Qld and } \\
\text { northern SA }\end{array}$
\end{tabular}

slander people

Occupational groups

Consider only in workers with a high risk of exposure to drug-resistant TB

Travellers

Recommended for child travellers at increased risk of acquiring TB, such as those:

- $<5$ years of age

- travelling to a high-incidence country ( $>40$ cases of TB per 100,000 population/year)

- with a cumulative duration of travel to a high incidence area of $>1$ month during early childhood

People with exposure to Hansen's disease

Recommended for young children and household contacts of people who have Hansen's disease

$T B$, tuberculosis

Investigation for LTBI (using a TB IGRA) and, if positive, consideration of treatment for LTBI is the preferred way of managing contacts of those with TB. Such investigations should be directed by state or territory jurisdictional TB programs. ${ }^{10}$

There have been shortages of BCG vaccine in recent years. The strain registered for use in Australia has been unavailable or subject to supply interruptions since 2012. ${ }^{11}$ There are limited supplies of other BCG vaccine strains available for which special prescribing arrangements are required. As a result of these shortages, it is important to prioritise vaccine access to those who will be benefit most from vaccination.

Where BCG vaccination is indicated, referral to a specialist TB or travel medicine centre with access to the BCG vaccine may be required in some jurisdictions. Table 3 includes contact information for state- and territoryspecific TB services.

\section{Efficacy}

There is wide variability in the reported efficacy of the BCG vaccine in preventing TB. This partly stems from the different outcomes used (TB disease versus TB infection as measured with an IGRA or a TST), and partly from the difference in efficacy between subgroups. Some of the variability might also be attributable to different BCG vaccine strains ${ }^{4}$ and exposure to other environmental mycobacteria. ${ }^{12}$ The greatest benefit of BCG vaccination is in the prevention of severe disseminated disease in young children.

Estimates of the efficacy of BCG vaccination in preventing adult pulmonary disease vary widely in different settings $(0-80 \%),{ }^{5}$ with an average protection rate of $50 \% .^{3}$ A meta-analysis of BCG trials showed a relative risk of 0.49 (95\% confidence interval: $0.34,0.64)$ for $\mathrm{TB}$ disease in those who had received the BCG vaccine. ${ }^{13}$

The availability of TB IGRAs has provided an opportunity to consider whether the BCG vaccine provides protection against $\mathrm{TB}$ acquisition rather than simply reducing risk of progression to active disease. A meta-analysis of studies 
of TB IGRA positivity in children who were recently exposed to TB found a protective efficacy of $19 \%$ in BCG-vaccinated children. ${ }^{14}$ The same study looked at progression to active disease in exposed children and found a protective effect of $71 \%$ against active disease; of those infected, there was a protective effect of $58 \%$ against progression to active disease. ${ }^{14}$

The BCG vaccine's protective effect against TB meningitis and miliary TB is consistently estimated at between $70 \%$ and $90 \% .{ }^{14,6}$ This may be due to the younger age of people usually affected by TB meningitis or miliary TB and waning of the vaccine's protective effect, or due to a different pathogenesis of pulmonary disease. BCG vaccination has been shown to be highly cost effective for children in high-incidence TB settings. ${ }^{6}$

\section{Other uses and off-target effects}

BCG vaccination also provides protection against nontuberculous mycobacteria. BCG vaccination protects against Hansen's disease, caused by $M$. leprae, with an overall protective effect of approximately $25 \% .{ }^{15}$ There are also observational data from European studies suggesting that BCG vaccination provides some protection against nontuberculous mycobacterial lymphadenitis in children. ${ }^{16}$ Of interest to those living in south-eastern Australia, the BCG vaccine is not thought to provide significant protection against $M$. ulcerans infection, although it may protect against M. ulcerans osteomyelitis. ${ }^{15,17,18}$

BCG vaccination also has 'off-target' effects beyond preventing TB. ${ }^{19}$ In the Australian context, the BCG vaccine is most recognised for its use in treating bladder cancer. In this situation, it is administered through multiple intravesicular instillations and acts as an immunomodulatory agent. ${ }^{20}$ The preparation of BCG vaccine for this purpose contains roughly 1000-fold the concentration of organisms in a BCG vaccine used for TB prevention, and serious adverse effects can occur if administered inappropriately.

It has also been reported from randomised controlled trials conducted in settings with high infant mortality that BCG vaccination provides survival benefit for infants independent of its TB protective effect. BCG vaccination has been shown to reduce all-cause neonatal mortality by preventing deaths from sepsis and rates of respiratory infections other than pulmonary TB. ${ }^{21}$ This off-target (or 'non-specific') effect of BCG vaccination is attributed to the ability of this vaccine to induce trained immunity in the innate immune system. ${ }^{22}$

There is a range of exploratory uses of $B C G$ vaccine currently under investigation in Australia. A trial of BCG vaccine for the treatment of multifocal motor neuropathy and multifocal acquired demyelinating sensory and motor neuropathy is underway (ACTRN12618001936213). Topically, there has also been interest in the role of $B C G$ vaccination in protecting vulnerable populations from severe COVID-19 disease. It has been postulated that the effect of BCG vaccination on innate immunity may reduce SARS-CoV-2 viraemia following exposure, with subsequent lessened risk of severe COVID-19 disease..$^{23}$ This hypothesis is being investigated in a number of trials internationally, including the Australian 'BCG vaccination to Reduce the impact of COVID-19 in Australian healthcare workers following Coronavirus Exposure' (BRACE) trial (NCT04327206).

\section{Table 3. State- and territory-specific tuberculosis services}

\begin{tabular}{|c|c|}
\hline State/territory & Tuberculosis service \\
\hline Australian Capital Territory & $\begin{array}{l}\text { Canberra Hospital Respiratory and Sleep Medicine, http://findahealthservice.act.gov.au/c/ } \\
\text { fahs } ? a=s p \& p i d=1316133581 \& \text { site }=52990 \& \text { servicecategory }=28\end{array}$ \\
\hline New South Wales & NSW Health - Tuberculosis, www.health.nsw.gov.au/Infectious/tuberculosis/Pages/default.aspx \\
\hline Northern Australia & $\begin{array}{l}\text { Darwin Tuberculosis and Leprosy Unit, https://nt.gov.au/wellbeing/health-conditions-treatments/bacterial/ } \\
\text { tuberculosis-tb/tb-clinic }\end{array}$ \\
\hline Queensland & $\begin{array}{l}\text { Queensland Health - Contact a tuberculosis service, www.health.qld.gov.au/clinical-practice/guidelines- } \\
\text { procedures/diseases-infection/diseases/tuberculosis/contact-a-service }\end{array}$ \\
\hline South Australia & $\begin{array}{l}\text { SA Health - SA Tuberculosis Services in Central Adelaide Local Health Network, } \\
\text { www.sahealth.sa.gov.au/wps/wcm/connect/public+content/sa+health+internet/services/hospitals/ } \\
\text { outpatient+services/outpatient+clinics/central+adelaide+lhn+specialist+and+outpatient+clinics/ } \\
\text { sa+tuberculosis+services+in+calhn }\end{array}$ \\
\hline Victoria & $\begin{array}{l}\text { The Royal Melbourne Hospital - Victorian Tuberculosis Program, www.thermh.org.au/health-professionals/ } \\
\text { clinical-services/victorian-tuberculosis-program }\end{array}$ \\
\hline Western Australia & $\begin{array}{l}\text { Department of Health - Western Australia Tuberculosis Control Program, https://ww2.health.wa.gov.au/ } \\
\text { Articles/A_E/About-the-Western-Australia-Tuberculosis-Control-Program }\end{array}$ \\
\hline
\end{tabular}


While clinical trials for novel indications are critical, the efficacy of BCG vaccination for these conditions remains uncertain. Because of the potential side effects and limited access to the vaccine, BCG vaccination should not be used for these indications outside of clinical trials. ${ }^{23}$

\section{Adverse effects and contraindications}

The BCG vaccine is generally safe and well tolerated. Most people who receive the BCG vaccine experience mild local reactions at the injection site, ${ }^{1}$ with serious adverse effects being rare. The rate of adverse events following immunisation in a study conducted in Victoria was approximately 15 per 10,000 doses administered. ${ }^{24}$ The most common adverse reactions are injection site abscesses (which may be prolonged in some cases and require local wound care), lymphadenitis and severe local reactions. ${ }^{25}$ Osteomyelitis can occur, likely through haematogenous spread, and can be difficult to treat. ${ }^{26}$ Disseminated BCG disease can occur, almost exclusively in patients who are severely immunocompromised. ${ }^{25-27}$

BCG vaccination should be undertaken by practitioners with appropriate training in administration and adverse effect monitoring.

There are multiple contraindications to BCG vaccine administration (Table 4). It is a live vaccine and therefore should not be administered to individuals who are immunocompromised. This includes people living with HIV infection ${ }^{28}$ and infants born to mothers treated with biological diseasemodifying antirheumatic drugs in the third trimester of pregnancy. ${ }^{1}$ BCG vaccination should also not be given to those who have had TB previously or those with a large reaction to the TST. $^{1}$

There are also some groups for whom precaution should be exercised when administering the BCG vaccine. These include unwell infants, people with a significant febrile illness and people with skin disease at the site of injection. People on treatment for LTBI should not receive the BCG vaccine as the treatment is likely to inactivate the vaccine. Infants born to mothers living with HIV should be shown to be HIV negative prior to receiving the BCG vaccine.

\section{Conclusion}

Even in the era of SARS-CoV-2, TB is the leading infective cause of death worldwide. The BCG vaccine is a safe, effective and cost-effective method of preventing many TB-related deaths, especially in young children. While it is important to investigate and understand the role of the BCG vaccine in other areas, this should not be at the expense of its proven use for TB protection. Further evidence on safety and efficacy is required to assess the role of BCG vaccination for indications other than TB prevention.

\section{Table 4. Contraindications to Bacille Calmette-Guérin vaccination}

\begin{tabular}{ll}
\hline Absolute contraindications & Relative contraindications \\
\hline - Individuals who are immunocompromised, & - Unwell infants \\
including those with & - Current febrile illness \\
- HIV & - Skin disease at proposed site of infection \\
- biological disease-modifying & - Current treatment for LTBI \\
$\quad$ antirheumatic drugs (and infants born & - Infants born to mothers living with HIV, \\
$\quad$ to mothers treated with same during & unless shown to be HIV negative \\
the third trimester of pregnancy) & \\
- other significant immunocompromise & \\
- Previous TB infection & \\
- Large reaction to TST & \\
\hline
\end{tabular}

HIV, human immunodeficiency virus; LTBI, latent tuberculosis infection; TB, tuberculosis; TST, tuberculin skin test

\section{Authors}

Jemma Wittner Taylor BBNSc, MBBS, DTMH, Infectious Diseases Advanced Trainee, The Royal Melbourne Hospital, Vic

Nigel Curtis MA, MBBS, DCH, DTM\&H, FRCPCH, PhD, Professor of Paediatric Infectious Diseases, Department of Paediatrics, The University of Melbourne, Vic; Leader of Infectious Diseases Group, Murdoch Children's Research Institute, Vic; Head of Infectious Diseases Unit, The Royal Children's Hospital Melbourne, Vic Justin Denholm BMed, MBioethics, MPH+TM, PhD, FRACP, Medical Director, Victorian Tuberculosis Program, Vic; Principle Investigator, Doherty Institute, The University of Melbourne, Vic. justin.denholm@mh.org.au

Competing interests: NC is the Chief Principal Investigator of the 'BCG vaccination to Reduce the impact of COVID-19 in Australian healthcare workers following Coronavirus Exposure' (BRACE) trial (NCT04327206).

Funding: None.

Provenance and peer review: Commissioned, externally peer reviewed.

\section{References}

1. Australian Immunisation Handbook. Tuberculosis. Canberra, ACT: DoH, 2018. Available at https:// immunisationhandbook.health.gov.au/vaccinepreventable-diseases/tuberculosis [Accessed 13 August 2020].

2. Luca S, Mihaescu T. History of BCG vaccine. Maedica (Buchar) 2013;8(1):53-58.

3. Hussey G, Hawkridge T, Hanekom W. Childhood tuberculosis: Old and new vaccines. Paediatr Respir Rev 2007;8(2):148-54. doi: 10.1016/j. prrv.2007.04.009.

4. Ritz N, Hanekom WA, Robins-Browne R, Britton WJ, Curtis N. Influence of BCG vaccine strain on the immune response and protection against tuberculosis. FEMS Microbiol Rev 2008;32(5):821-41. doi: 10.1111/j.15746976.2008.00118.x.

5. Fine PEM, Carneiro IAM, Milstien JB, Clements CJ. Issues relating to the use of BCG in immunization programmes: A discussion document. Geneva, $\mathrm{CH}:$ WHO, 1999.

6. Trunz BB, Fine P, Dye C. Effect of BCG vaccination on childhood tuberculous meningitis and miliary tuberculosis worldwide: A meta-analysis and assessment of cost-effectiveness. Lancet 2006;367(9517):1173-80. doi: 10.1016/S01406736(06)68507-3.

7. Gwee A, Rodrigo R, Casalaz D, Ritz N, Curtis N Infants born in Australia to mothers from countries with a high prevalence of tuberculosis: To BCG or not to BCG? Med J Aust 2014;200(3):149-50. doi: 10.5694/mja13.11367.

8. National Tuberculosis Advisory Committee. The BCG vaccine: Information and recommendations for use in Australia. Commun Dis Intell 2006;30(1):109-15.

9. Ritz N, Connell TG, Curtis N. To BCG or not to BCG? Preventing travel-associated tuberculosis in children. Vaccine 2008;26(47):5905-10. doi: $10.1016 /$ j.vaccine.2008.08.061.

10. Stock D. National position statement for the management of latent tuberculosis infection. Commun Dis Intell Q Rep 2017;41(3):E204-08.

11. Khandaker G, Beard FH, Dey A, Coulter C, Hendry AJ, Macartney KK. Evaluation of Bacille Calmette-Guérin immunisation programs in Australia. Commun Dis Intell Q Rep 2017;41(1):E33-E48. 
12. Fine PE. Variation in protection by BCG: Implications of and for heterologous immunity. Lancet;346(8986):1339-45. doi: 10.1016/s01406736(95)92348-9.

13. Colditz GA, Brewer TF, Berkey CS, et al. Efficacy of BCG vaccine in the prevention of tuberculosis: Meta-analysis of the published literature. JAMA 1994;271(9):698-702. doi: 10.1001/ jama.1994.03510330076038.

14. Roy A, Eisenhut M, Harris RJ, et al. Effect of BCG vaccination against Mycobacterium tuberculosis infection in children: Systematic review and metaanalysis. BMJ 2014;349:g4643. doi: 10.1136/bmj. g4643.

15. Setia MS, Steinmaus C, Ho CS, Rutherford GW. The role of BCG in prevention of leprosy: A metaanalysis. Lancet Infect Dis 2006;6(3):162-70. doi: 10.1016/S1473-3099(06)70412-1.

16. Zimmermann P, Finn A, Curtis N. Does BCG vaccination protect against nontuberculous mycobacterial infection? A systematic review and meta-analysis. J Infect Dis 2018;218(5):679-87. doi: 10.1093/infdis/jiy207.

17. Phillips RO, Phanzu DM, Beissner M, et al. Effectiveness of routine BCG vaccination on Buruli ulcer disease: A case-control study in the Democratic Republic of Congo, Ghana and Togo. PLoS Negl Trop Dis 2015:9(1):e3457. doi: 10.1371/ journal.pntd.0003457.

18. Portaels F, Aguiar J, Debacker M, et al. Mycobacterium bovis BCG vaccination as prophylaxis against Mycobacterium ulcerans osteomyelitis in Buruli ulcer disease. Infect Immun 2004;72(1):62-65. doi: 10.1128/iai.72.1.62-65.2004.

19. Pollard AJ, Finn A, Curtis N. Non-specific effects of vaccines: Plausible and potentially important, but implications uncertain. Arch Dis Child 2017;102(11):1077-81. doi: 10.1136/ archdischild-2017-314182.

20. Prescott S, Jackson AM, Hawkyard SJ, Alexandroff $A B$, James K. Mechanisms of action of intravesical Bacille CalmetteGuérin: Local immune mechanisms. Clin Infect Dis 2000;31(Supplement 3):S91-93. doi: 10.1086/314066.

21. Biering-Sørensen S, Aaby P, Lund N, et al. Early BCG-Denmark and neonatal mortality among infants weighing <2500 g: A randomized controlled trial. Clin Infect Dis 2017;65(7):1183-90. doi: 10.1093/cid/cix525.

22. Netea MG, Domínguez-Andrés J, Barreiro LB, et al. Defining trained immunity and its role in health and disease. Nat Rev Immunol 2020;20(6):375-88. doi: 10.1038/s41577-020-0285-6.

23. Curtis N, Sparrow A, Ghebreyesus TA, Netea MG. Considering BCG vaccination to reduce the impact of COVID-19. Lancet 2020;395(10236):1545-46. doi: 10.1016/S01406736(20)31025-4.

24. Clothier HJ, Hosking L, Crawford NW, et al. Bacillus Calmette-Guérin (BCG) vaccine adverse events in Victoria, Australia: Analysis of reports to an enhanced passive surveillance system. Drug Saf 2015;38(1):79-86. doi: 10.1007/s40264-0140248-6.

25. Turnbull FM, Mclntyre PB, Achat HM, et al. National study of adverse reactions after vaccination with Bacille CalmetteGuérin. Clin Infect Dis 2002;34(4):447-53. doi: $10.1086 / 338462$

26. Huang CY, Chiu NC, Chi H, Huang FY, Chang PH. Clinical manifestations, management, and outcomes of osteitis/osteomyelitis caused by Mycobacterium bovis Bacillus Calmette-Guérin in children: Comparison by site(s) of affected bones. J Pediatr 2019;207:97-102. doi: 10.1016/j. jpeds.2018.11.042

27. Murphy D, Corner LAL, Gormley E. Adverse reactions to Mycobacterium bovis Bacille Calmette-Guérin (BCG) vaccination against tuberculosis in humans, veterinary animals and wildlife species. Tuberculosis (Edinb) 2008;88(4):344-57. doi: 10.1016/j.tube.2007.11.010.

28. Hesseling AC, Marais BJ, Gie RP, et al. The risk of disseminated Bacille Calmette-Guerin (BCG) disease in HIV-infected children. Vaccine 2007;25(1):14-18. doi: 10.1016/j. vaccine.2006.07.020. 